\title{
ERRATUM
}

Robin F. A. Moritz • H. Michael • G. Lattorff •

Peter Neumann - F. Bernhard Kraus •

Sarah E. Radloff · H. Randall Hepburn

\section{Rare royal families in honeybees, Apis melliferas}

Published online: 18 October 2005

(C) Springer-Verlag 2005

\section{Naturwissenschaften (2005) 92: 488-491}

The list of addresses was incorrect in the original publication.

The correct list is given here:

(1) Institut für Zoologie, Martin-Luther-Universität Halle-Wittenberg, 06099 Halle (Saale), Germany, E-mail: r.moritz@zoologie.uni-halle.de, Tel.:+49-345-5526223, Fax: $+49-345-5527264$

(2) Department of Statistics, Rhodes University, 6140 Grahamstown, South Africa

(3) Department of Zoology and Entomology, Rhodes University, 6140 Grahamstown, South Africa

(4) Present address: El Colegio de la Frontera Sur, Departamento Entomología Tropical, C.P. 30700, Tapachula, Chiapas, México

R. F. A. Moritz $(\bowtie) \cdot$ H. Michael · G. Lattorff · P. Neumann

F. B. Kraus

Institut für Zoologie,

Martin-Luther-Universität Halle-Wittenberg,

06099 Halle, Saale, Germany

e-mail: r.moritz@zoologie.uni-halle.de

Tel.: +49-345-5526223

Fax: +49-345-5527264

S. E. Radloff

Department of Statistics, Rhodes University,

6140 Grahamstown, South Africa

P. Neumann · H. R. Hepburn

Department of Zoology and Entomology, Rhodes University,

6140 Grahamstown, South Africa

Present Address

F. B. Kraus

El Colegio de la Frontera Sur,

Departamento Entomología Tropical,

C.P. 30700 , Tapachula, Chiapas, México 